\title{
ĐỊNH VỊ ĐIỂM CHÍNH XÁC CAO DÙNG VỆ TINH GALILEO CÓ GIẢI ĐA TR!
}

\author{
NGUYẼ̃N NGỌC LÂU \\ Truờng Đại học Bách khoa TP.HCM
}

\section{Tóm tắt:}

Tù tuần lễ GPS 2034, Trung tâm Nghiên cưu Không gian Quốc gia Pháp bắt đầu cung cấp sản phẩm chính xác bản lịch và số hiệu chỉnh đồng hồ vệ tinh GALILEO có hố trợ cho việc giải đa trị. Chúng tôi đã khảo sát độ chính xác định vị điểm chỉ dùng GALILEO có giải đa trị tại 12 trạm GNSS thường trục ở khu vực Châu Âu. Kết quả cho thấy độ chính xác khi xử lý tĩnh 24 hà $(2.4,3.8,7.4) m m$ và khi xủ lý động $(9.7,8.8,26.8) m m$ theo hướng Bắc, Đông và độ cao. So với độ chính xác GPS tĩnh, thì thành phần hướng Đông và độ cao của GALILEO vẫn kém hơn gần 1/2. Trong khi đó độ chính xác định vị động GALILEO lại không có sụ chênh lệch nhiều so với GPS.

\section{Giới thiệu}

GALILEO là hệ thống vệ tinh định vị toàn cầu do Châu Âu xây dựng và phát triển, tương tự như GPS của Mỹ và GLONASS của Nga. Vệ tinh thử nghiệm đầu tiên GIOVE $\mathrm{A}$ đã được phóng từ 2005. Hiện nay số lượng vệ tinh GALILEO hoạt động và đang phát tín hiệu trên quỹ đạo là 23. Với số lượng vệ tinh này, đa số các khu vực trên thế giới có thể quan sát từ 4 vệ tinh GALILEO trở lên và có thể thực hiện định vị một cách độc lập. Tuy nhiên con số này vẫn chỉ đạt $\sim 2 / 3$ so với thiết kế sẽ có 30 vệ tinh hoạt động [1]. (Xem bảng 1)

Trong số các hệ thống định vị toàn cầu hiện có, GALILEO có nhiều điểm tương tự nhất với GPS về

- Phương pháp mã hóa tín hiệu CDMA

- Tần số E1 giống với L1 GPS là 1575.42
$\mathrm{MHz}$, nhưng khác tần số thứ 2 dùng cho mục đích dân sự E5a $1176.45 \mathrm{MHz}$

- Có hệ thống thời gian và hệ thống tọa độ gần như trùng nhau

Do đó sẽ có nhiều thuận lợi khi xử lý riêng biệt GALILEO và xử lý tích hợp GPS+GALILEO.

Trung tâm Nghiên cứu Không gian Quốc gia Pháp (Centre National d'Etudes Spatiales CNES) đã cung cấp sản phẩm bản lịch và số hiệu chỉnh đồng hồ vệ tinh GPS có hỗ trợ cho việc giải đa trị từ rất sớm [9]. Nhờ đó mà định vị điểm chính xác cao có giải đa trị (Precise Point Positioning with Ambiguity Resolution - PPP $\mathrm{AR}$ ) dùng GPS đã đạt được những thành tựu mới về cải thiện độ chính xác [5]. Đặc biệt bắt đầu từ tuần lễ GPS 2034 (30-12-2018), trung tâm đã cung cấp thêm sản phẩm tương tự có hỗ trợ giải đa trị cho GALILEO. Điều này mở ra khả năng

Bảng 1: Trạng thái của GALILEO vào 6/2020 (tham khảo tù̀ [2])

\begin{tabular}{|c|l|l|l|l|l|}
\hline \multirow{2}{*}{ Block } & \multirow{2}{*}{ Thời gian phóng } & \multicolumn{3}{|c|}{ Trạng thái phóng } & \multirow{2}{*}{$\begin{array}{c}\text { Hoạt động vàn } \\
\text { khỏe mạnh }\end{array}$} \\
\cline { 3 - 5 } & Thành công & Thất bại & Dụ̂ kiến & \\
\hline GIOVE & $2005-2008$ & 2 & 0 & 0 & 0 \\
\hline IOV & $2011-2012$ & 4 & 0 & 0 & 3 \\
\hline FOC & From 2014 & 20 & 2 & 12 & 20 \\
\hline & Tống cộng & 26 & 2 & 12 & 23 \\
\hline
\end{tabular}

Ngày nhận bài: 25/10/2020, ngày chuyển phản biện: 29/10/2020, ngày chấp nhận phản biện: 05/11/2020, ngày chấp nhận đăng: 09/11/2020 
nâng cao độ chính xác cho định vị điểm trị đo pha GALILEO khi giải tham số đa trị và sự kết hợp giữa GPS và GALILEO.

Ngay sau sự kiện này, một vài nhà khoa học đã khảo sát và đánh giá độ chính xác định vị điểm GALILEO với việc giải đa trị dùng sản phẩm của CNES. Katsigianni và nnk [3] đã xử lý động dữ liệu GNSS của khoảng 50 trạm IGS thường trực. Kết quả cho thấy độ chính xác PPP$\mathrm{AR}$ chỉ dùng GALILEO là cùng cấp với GPS only và đạt được tốt nhất ở khu vực Châu Âu (thử nghiệm tại 3 trạm) với 2-4 mm ở mặt bằng, $10 \mathrm{~mm}$ ở độ cao. Ở mức độ toàn cầu, độ chính xác chỉ đạt $1 \mathrm{~cm}$ ở mặt bằng và $3 \mathrm{~cm}$ ở độ cao. Trong bài báo [4], Katsigianni và nnk [4] đã mở rộng nghiên cứu xử lý động dữ liệu GNSS tại 4 trạm ở Châu Âu. Kết quả độ chính xác định vị trung bình $7 \mathrm{~mm}$ ở mặt bằng và $28 \mathrm{~mm}$ ở độ cao. Tuy nhiên những kết quả đạt được này có thể chưa khách quan và chính xác khi các tác giả dùng số trạm đo quá ít ở khu vực Châu Âu. Hơn nữa độ chính xác định vị không những chỉ phụ thuộc vào vị trí mà còn phụ thuộc vào những yếu tố quan trọng khác như chât lượng máy thu và anten.

Trong bài báo này chúng tôi sẽ thực hiện việc khảo sát trên mức độ toàn cầu của trạng thái GALILEO để xem khu vực nào có thể đạt được độ chính xác cao nhất. Và trên khu vực ấy chúng tôi sẽ xử lý số lượng trạm đo nhiều hơn để có thể đạt được kết quả đánh giá độ chính xác định điểm khi đo tĩnh và động thực tế hơn.

\section{Bộ dữ liệu GNSS dùng trong khảo sát}

Mạng lưới các trạm IGS thường trực hiện có hơn 300 trạm phân bố toàn cầu. Tại các trạm này được trang bị các máy thu GNSS đa hệ thống vệ tinh có thể thu tín hiệu từ GPS và cả GALILEO. Đây là nguồn dữ liệu tốt để dùng cho việc khảo sát. Tuy nhiên, hiện nay số lượng các vệ tinh GALILEO trên quĩ đạo vẫn chưa đầy đủ (23/30). Do đó số lượng vệ tinh GALILEO được quan sát ở mỗi vị trí khác nhau trên quả đất sẽ không giống nhau. Để đánh giá độ chính xác định vị điểm dùng trị đo GALILEO, ta nên thực hiện ở khu vực có số lượng vệ tinh GALILEO bao phủ nhiều nhất. Khu vực này có thể là Châu Âu và cũng có thể là một nơi nào đó.

Để tìm ra khu vực thích hợp nêu trên, chúng tôi thực hiện việc lấy mẫu ở một số nơi khác nhau trên bề mặt trái đất vào ngày 16-06-2020. Việc lấy mẫu là tìm số lượng GALILEO quan sát được tại vị trí đó trong ngày. Và vì chu kỳ chuyển động của các vệ tinh GALILEO xấp xỉ $12 \mathrm{~h}$ nên vị trí của các vệ tinh này sẽ gần như lặp lại cho những ngày tiếp theo. (Xem hình 1)

Hình 1 thể hiện đồ thị số lượng vệ tinh GPS và GALILEO quan sát được ở mỗi vị trí trong thời gian 24h ngày 16-06-2020. Các trạm đo lấy mẫu là DARW (Úc), BRUX (Châu Âu), CUSV (Đông Nam Á), WUH2 (Trung Quốc), GODE (Bắc Mỹ), và SANT (Nam Mỹ). Các đồ thị này cho thấy tại tất cả các trạm đo số lượng vệ tinh trung bình của GALILEO đều lớn hơn 4 nhưng luôn nhỏ hơn số lượng GPS. Trạm có số lượng vệ tinh trung bình GALILEO cao nhất là BRUX ở Châu Âu (8-9 vệ tinh). Như vậy, việc định vị chỉ dùng vệ tinh GALILEO là có thể thực hiện được tại mọi nơi. Khu vực có độ chính xác cao nhất vẫ் là Châu Âu.

Dựa vào phân tích trên, chúng tôi chọn ra 12 trạm GNSS thường trực phân bố trên khu vực Châu Âu. Những trạm này đều được trang bị các máy thu GNSS đa hệ thống vệ tinh có tốc độ thu 30s. Các máy thu được chọn khác loại để đảm bảo tính khách quan trong khảo sát (xem bảng 2)

Tọa độ chính xác của các trạm này được lấy từ việc bình sai mạng lưới IGS toàn cầu hàng tuần với độ chính xác vài $\mathrm{mm}$ trong hệ tọa độ ITRF2014 [8].

\section{Phần mềm và kết quả xử lý PPP}

Để xử lý định vị điểm chính xác cao dữ liệu GNSS của 12 trạm IGS ở bảng 2 , chúng tôi dùng phần mềm PPPC do chúng tôi tự phát triển từ 2010 [6]. Sau nhiều lần nâng cấp, phần mềm PPPC có khả năng xử lý dữ liệu GNSS ở cả hai chế độ tĩnh và động và cho nhiều hệ thống vệ tinh khác nhau như GPS, GLONASS, 


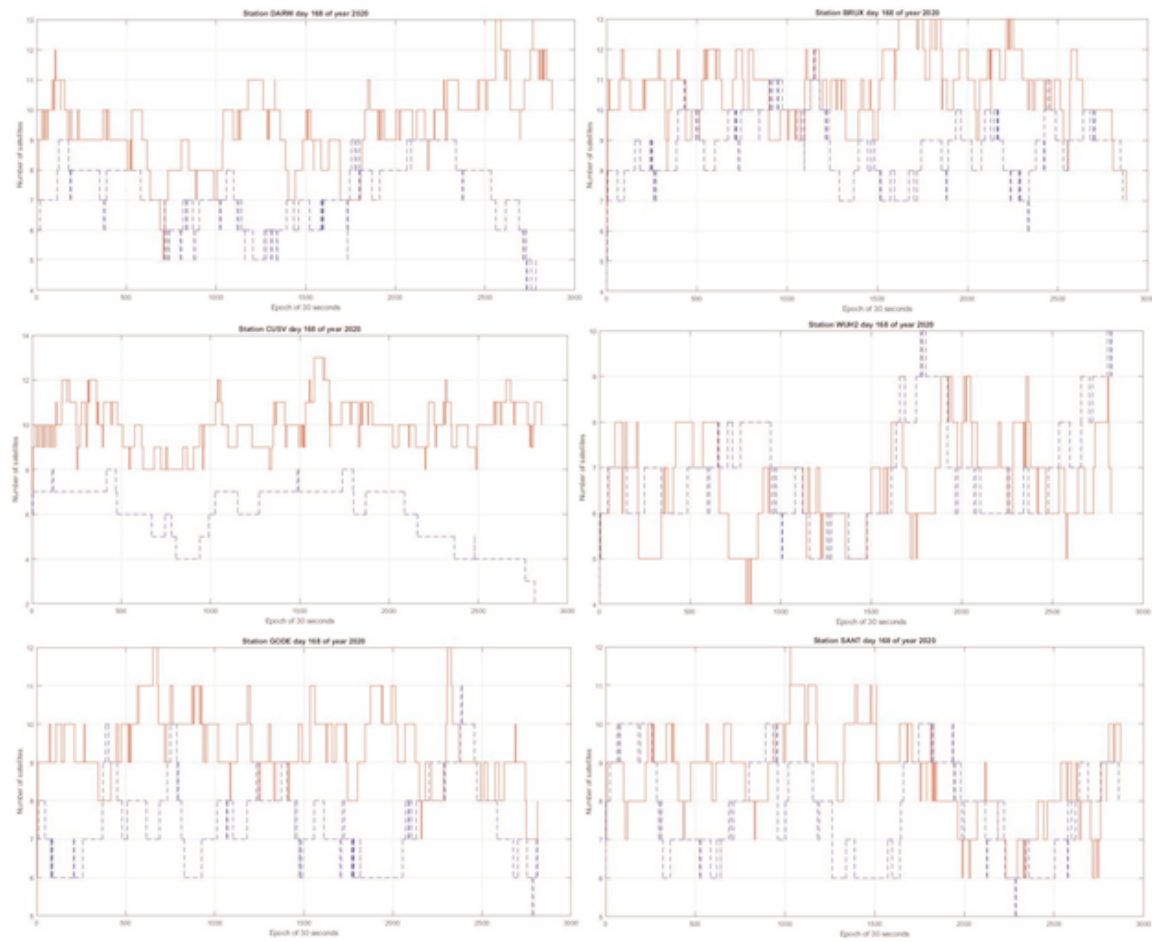

Hình 1: Trạng thái của vệ tinh GPS (liền nét đỏ) và GALILEO (đứt nét xanh) quan sát tại một số các vị trí khác nhau

Bảng 2: Các trạm GNSS thường trục dùng trong khảo sát

\begin{tabular}{|c|c|c|c|c|}
\hline STT & Tên & Máy thu & Loại anten & Quóc gia \\
\hline 1 & AJAC & SEPT POLARX5 & TRM115000.00 NONE & France \\
\hline 2 & ANKR & LEICA GR30 & LEIAR10 NONE & Turkey \\
\hline 3 & CEBR & SEPT POLARX5TR & SEPCHOKE_B3E6 NONE & Spain \\
\hline 4 & BRST & TRIMBLE ALLOY & TRM57971.00 NONE & France \\
\hline 5 & BRUX & SEPT POLARX5TR & JAVRINGANT_DM NONE & Belgium \\
\hline 6 & DYNG & TRIMBLE NETR9 & TRM59800.00 NONE & Greece \\
\hline 7 & HOFN & LEICA GR50 & LEIAR25.R4 LEIT & Iceland \\
\hline 8 & HUEG & JAVAD TRE_3 DELTA & LEIAR25.R4 & Germany \\
\hline 9 & KIRU & SEPT POLARX5 & SEPCHOKE_B3E6 SPKE & Sweden \\
\hline 10 & KRGG & TRIMBLE ALLOY & LEIAR25.R4 & France \\
\hline 11 & METG & TRIMBLE NETR9 & TRM59800.00 SCIS & Finland \\
\hline 12 & QAQ1 & SEPT POLARX5 & ASH701945E_M SCIS & Greenland \\
\hline
\end{tabular}

GALILEO, BEIDOU và QZSS. Gần đây nhất chúng tôi đã nâng cấp PPPC thêm khả năng giải đa trị dùng sản phẩm IRC của CNES. Việc khảo sát độ chính xác định vị điểm chỉ dùng GPS có giải đa trị đã được chúng tôi thực hiện trong bài báo [5]. Để xử lý GALILEO có giải đa trị, chúng tôi áp dụng cách thức tương tự như GPS [5]. (Xem bảng 3)

Một số cài đặt chung cho quá trình xử lý được cho ở Bảng 3. Dũ̃ liệu GNSS ngày 16-06-2020 của mỗi trạm đo được xử lý 4 phương án như sau:

- Xử lý tĩnh 24h chỉ dùng trị đo GPS

- Xử lý tĩnh 24h chỉ dùng trị đo GALILEO

- Xử lý động chỉ dùng trị đo GPS

- Xử lý động chỉ dùng trị đo GALILEO 
Các tọa độ nhận được từ việc xử lý được chuyển sang thành phần hướng Bắc, hướng Đông và độ cao, rồi trừ đi với giá trị chính xác của nó để tính ra hiệu tọa độ $\Delta \mathrm{N}_{\mathrm{i}}, \Delta \mathrm{E}_{\mathrm{i}}$, và $\Delta \mathrm{U}_{\mathrm{i}}$ cho từng trạm đo $\mathrm{i}$ trong xử lý tĩnh hay từng thời điểm i trong xử lý động. Các hiệu tọa độ này sau đó được dùng để tính ra sai số trung phương theo công thức:

$$
m_{N}=\sqrt{\frac{\sum_{i=1}^{n} \Delta N_{i}^{2}}{n}} ; m_{E}=\sqrt{\frac{\sum_{i=1}^{n} \Delta E_{i}^{2}}{n}} ; m_{U}=\sqrt{\frac{\sum_{i=1}^{n} \Delta U_{i}^{2}}{n}}
$$

Kết quả xử lý tĩnh $24 \mathrm{~h}$ của GPS và GALILEO được cho ở Bảng 4. Kết quả xử lý động cho ở Bảng 5 . Khi xử lý GPS, tỷ lệ giải thành công tham số đa trị đạt rất cao, trung bình là $96 \%$. Đối với GALILEO, tỷ lệ này thấp hơn, đạt trung bình 90\%. (Xem bảng 4, 5)

Bảng 4 và 5 cho thấy độ chính xác định vị dùng trị đo GALILEO luôn thấp hơn so với dùng trị đo GPS. Điều này có thể do số lượng vệ tinh GALILEO quan sát được tại mỗi trạm đo luôn thấp hơn GPS. Trong tương lai khi GALILEO có đầy đủ số lượng vệ tinh, độ chính xác này sẽ được cải thiện hơn nữa và tương đương với GPS.

Độ chính xác định vị tĩnh 24h của GALILEO chỉ tương đương với GPS ở thành phần hướng Bắc (2.4mm so với $2.8 \mathrm{~mm})$, còn hướng Đông và độ cao thì kém gấp đôi $(3.8 \mathrm{~mm} 7.4 \mathrm{~mm}$ so với
$1.3 \mathrm{~mm} 3.3 \mathrm{~mm}$ ). Điều tương tự cũng quan sát thấy trên kết quả xử lý động, nhưng sự thua kém ở thành phần hướng Đông và độ cao ít hơn nhiều. Nhìn chung độ chính xác định vị động dùng GPS hay GALILEO xấp xỉ $1 \mathrm{~cm}$ ở mặt bằng và $2-3 \mathrm{~cm}$ ở thành phần độ cao.

\section{So sánh với các nghiên cứu khác}

Katsigianni và nnk [3] xử lý định vị động dữ liệu GNSS của 3 trạm BRUX, CAS1 và NYA2 trong 7 ngày 042-048 của năm 2019. Họ dùng giá trị trung bình của tọa độ trạm đo xử lý trong 7 ngày này để làm cơ sở so sánh, và tính ra được sai số trung phương định vị theo hướng Đông, hướng Bắc và độ cao. Độ chính xác đạt được khi xử lý GALILEO là $(2.6-4.2,2.9-5.2,10.3-$ $15.6 \mathrm{~mm})$ với GPS là $(2.4-3.8,2.2-5.2,8.5$ $14.3 \mathrm{~mm})$. Khi mở rộng xử lý khoảng 50 trạm đo toàn cầu, độ chính xác đạt được xấp xỉ $1 \mathrm{~cm}$ ở mặt bằng và $3 \mathrm{~cm}$ ở độ cao. Trong tài liệu [4], Katsigianni và nnk mở rộng xử lý 4 trạm đo ở Châu Âu đạt được độ chính xác khoảng $7 \mathrm{~mm}$ ở mặt bằng và $3 \mathrm{~cm}$ ở độ cao.

Kết quả của nhóm nghiên cứu Katsigiani nêu trên và kết quả xử lý động của chúng tôi ở bảng 5 là khá tương đồng với nhau. Mặc dù cả hai có sự khác biệt về tập dữ liệu và phương pháp tính toán.

Bảng 3: Các tham số cài đặt trong xủ lý PPP

\begin{tabular}{|c|c|}
\hline Nội dung & Giá trị \\
\hline Bản lịch và đồng hồ vệ tinh & Dùng sản phẩm CNES có hỗ trợ giài đa trị \\
\hline Mô hình anten & IGS2014 \\
\hline Trị đo & $\begin{array}{l}\text { Trị đo mã } P_{3} \text { và pha } \Phi_{3} \text { của } \\
\text { GPS/GALILEO ở dạng hiệu đơn giữa các } \\
\text { vệ tînh }\end{array}$ \\
\hline Góc cao vệ tinh giới hạn & $5^{\circ}$ \\
\hline Trọng số trị đo & $\operatorname{Exp}\left(-\varepsilon / 9^{\circ}\right), \varepsilon$ là góc cao vệ tính \\
\hline Độ trễ đối lưu & $\begin{array}{l}\text { Khào sát } 1 \text { tham số cho độ trễ thiên đỉnh } \\
\text { mỗi } 30 \text { phút và } 2 \text { tham số gradient mỗi } 12 \\
\text { giờ }\end{array}$ \\
\hline Hàm ánh xạ đối lưu & VMF3 [7] \\
\hline Giải đa trị & tham số đa trị giải rộng trước, giài hẹp sau \\
\hline $\begin{array}{l}\text { Hiệu chinh chuyển dịch trạm } \\
\text { đo do địa triều (solid Earth } \\
\text { tide) }\end{array}$ & Áp dụng \\
\hline $\begin{array}{l}\text { Hiệu chinh chuyển dịch trạm } \\
\text { đo do tải thùy triều (ocean } \\
\text { tide loading) }\end{array}$ & Áp dụng FES2012 \\
\hline
\end{tabular}


Bảng 4: Kết quả xủ lý tĩnh 24 h theo phưong án a và b

\begin{tabular}{|c|c|c|c|c|c|c|c|}
\hline \multirow{2}{*}{ STT } & \multirow{2}{*}{ Tên } & \multicolumn{3}{|c|}{ GPS (mm) } & \multicolumn{3}{c|}{ GALILEO (mm) } \\
\cline { 3 - 8 } & & $\Delta \mathrm{N}$ & $\Delta \mathrm{E}$ & $\Delta \mathrm{U}$ & $\Delta \mathrm{N}$ & $\Delta \mathrm{E}$ & $\Delta \mathrm{U}$ \\
\hline 1 & BRST & -4.4 & +3.1 & -3.9 & -0.5 & +6.4 & +2.7 \\
\hline 2 & BRUX & -0.7 & +0.3 & -2.4 & -0.6 & +4.8 & -11.0 \\
\hline 3 & KIRU & +0.5 & +2.0 & -1.9 & +4.4 & +4.0 & +5.1 \\
\hline 4 & KRGG & +1.8 & +1.9 & +3.9 & -2.0 & -2.2 & +9.0 \\
\hline 5 & QAQ1 & +2.0 & +2.6 & -5.7 & +0.7 & +3.1 & -5.6 \\
\hline 6 & AJAC & -1.7 & +0.8 & +0.9 & +2.5 & +2.1 & -4.2 \\
\hline 7 & ANKR & -4.4 & +1.4 & +4.4 & -2.7 & +2.3 & +9.7 \\
\hline 8 & CEBR & -3.7 & +2.8 & -4.4 & -2.1 & +4.5 & +2.6 \\
\hline 9 & DYNG & -1.1 & +1.4 & +2.5 & -0.1 & -0.7 & -8.1 \\
\hline 10 & HOFN & -0.3 & +5.1 & -3.3 & +0.1 & -5.5 & +3.4 \\
\hline 11 & HUEG & -4.7 & +2.3 & -1.4 & -1.4 & -4.5 & -8.0 \\
\hline 12 & METG & +3.7 & +1.0 & -1.3 & +4.5 & +2.7 & -10.0 \\
\hline \multicolumn{2}{|c|}{ SSTP } & 2.8 & 1.3 & 3.3 & 2.4 & 3.8 & 7.4 \\
\hline
\end{tabular}

Bảng 5: Kết quả xủ lý động theo phưong án c vàd

\begin{tabular}{|c|c|c|c|c|c|c|c|}
\hline \multirow{2}{*}{ STT } & \multirow{2}{*}{ Trạm } & \multicolumn{3}{|c|}{ GPS (mm) } & \multicolumn{3}{c|}{ GALILEO (mm) } \\
\cline { 3 - 8 } & & $\mathrm{m}_{\mathrm{N}}$ & $\mathrm{m}_{\mathrm{E}}$ & $\mathrm{m}_{\mathrm{U}}$ & $\mathrm{m}_{\mathrm{N}}$ & $\mathrm{m}_{\mathrm{E}}$ & $\mathrm{mU}$ \\
\hline 1 & BRST & 10 & 9 & 21 & 10 & 8 & 23 \\
\hline 2 & BRUX & 7 & 6 & 17 & 8 & 8 & 23 \\
\hline 3 & KIRU & 8 & 6 & 25 & 9 & 7 & 24 \\
\hline 4 & KRGG & 14 & 8 & 27 & 12 & 10 & 33 \\
\hline 5 & QAQ1 & 7 & 5 & 16 & 7 & 5 & 19 \\
\hline 6 & AJAC & 7 & 6 & 21 & 8 & 6 & 29 \\
\hline 7 & ANKR & 10 & 8 & 26 & 11 & 12 & 32 \\
\hline 8 & CEBR & 11 & 9 & 28 & 11 & 11 & 31 \\
\hline 9 & DYNG & 9 & 7 & 29 & 10 & 11 & 30 \\
\hline 10 & HOFN & 7 & 8 & 18 & 8 & 7 & 25 \\
\hline 11 & HUEG & 10 & 8 & 25 & 12 & 11 & 29 \\
\hline 12 & METG & 10 & 6 & 22 & 11 & 9 & 24 \\
\hline \multicolumn{2}{|c|}{ Trung bình } & 9.2 & 7.1 & 22.9 & 9.7 & 8.8 & 26.8 \\
\hline
\end{tabular}

\section{Kết luận}

Với cấu hình chưa hoàn chỉnh hiện nay, số lượng vệ tinh GALILEO quan sát được tại một vị trí bất kỳ trên bề mặt trái đất vẫn đủ cho việc định vị độc lập nhưng vẫn thua kém số lượng vệ tinh GPS. Điều này dẫn đến độ chính xác định vị chỉ dùng GALILEO vẫn chưa bằng với GPS. Khu vực có độ bao phủ GALILEO tốt nhất hiện nay vẫn là Châu Âu với số lượng vệ tinh quan sát trung bình 8-9. Đây cũng là khu vực mà chúng tôi chọn trong nghiên cứu này.

Nhờ vào sản phẩm bản lịch và số hiệu chỉnh đồng hồ vệ tinh GALILEO có hỗ trợ giải đa trị của CNES, độ chính xác định vị điểm khi xử lý tĩnh và động GALILEO đều được cải thiện và tiệm cận dần với độ chính xác GPS. Khảo sát của chúng tôi trên 12 trạm GNSS thường trực tại Châu Âu cho thấy độ chính xác khi xử lý tĩnh 24h là $(2.4,3.8,7.4) \mathrm{mm}$ và khi xử lý động $(9.7$, $8.8,26.8) \mathrm{mm}$ theo hướng Bắc, Đông và độ cao. So với độ chính xác GPS tĩnh, thì thành phần hướng Đông và độ cao của GALILEO vẫn kém hơn gần $1 / 2$. Trong khi đó độ chính xác động không chênh lệch nhiều.

Kết quả định vị động của chúng tôi tương đồng với kết quả nghiên cứu của nhóm Katsigiani $[4,5]$ mặc dù có sự khác biệt về tập dữ liệu khảo sát và phương pháp tính toán. $O$

\section{Tài liệu tham khảo}

[1]. European GNSS open service, (2018), "Signal-in-space Interface Control Document", 74 trang

[2]. Wikipedia, (2020), https://en.wikipedia.org/wiki/Galileo_(satellite_navigation). 
[3]. Katsigianni, G., Loyer, S. and Perosanz Felix, (2019), "PPP and PPP-AR Kinematic Post-Processed Performance of GPS-Only, Galileo-Only and Multi-GNSS". Remote Sens. 2019, 11(21).

[4]. Katsigianni, G., Perosanz, F., Loyer, S. et al., (2019), "Galileo millimeter-level kinematic precise point positioning with ambiguity resolution". Earth Planets Space 71, 76 (2019) doi:10.1186/s40623-019-1055-1.

[5]. Nguyễn Ngọc Lâu, (2017), “Độ chính xác PPP có giải tham số đa trị trong khung tọa độ mới ITRF2014", Hội nghị Khoa học và Công nghệ lần thứ 15 tổ chức tại ĐHBK TPHCM 10/2017, 47-54.

[6]. Nguyễn Ngọc Lâu, Trần Trọng Đức, Dương Tuấn Việt, Đặng Văn Công Bằng, (2010). "Automatic GPS precise point process- ing via internet”. Báo cáo đề tài cấp Bộ B201030-33, 107 trang.

[7]. Böhm, J., Werl, B., \& Schuh, H., (2006), "Troposphere mapping functions for GPS and VLBI from ECMWF operational analysis data". Journal of Geophysical Research, 111, B02406, doi:10.1029/2005JB003629.

[8]. Zuheir Altamimi, Paul Rebischung, Laurent Métivier, and Xavier Collilieux, (2016), "ITRF2014: A new release of the International Terrestrial Reference Frame modeling nonlinear station motions", Journal of Geophysical Research: Solid Earth, 121, pp 6109-6131, doi:10.1002/2016JB013098.

[9]. Laurichesse D., (2012), "Phase Biases Estimation for Undifferenced Ambiguity Resolution", PPP-RTK \& Open Standards Symposium, March 12-13, 2012, Frankfurt. $\bigcirc$

\section{Summary}

\section{Accuracy of precise point positioning with ambiguity resolution using galileo only}

Nguyen Ngoc Lau

Department of Geomatics Engineering, Ho Chi Minh City University of Technology

Vietnam National University Ho Chi Minh City

From GPS week 2034, the France Centre National d'Etudes Spatiales (CNES) started to provide accurate products of ephemerides and clock corrections for GALILEO satellites with ambiguity resolution capability. We investigated the accuracy of precise point positioning with ambiguity resolution using only GALILEO at 12 IGS permanent stations in the European region. The results show that the accuracy of 24-hour static solution is $(2.4,3.8,7.4) \mathrm{mm}$ and kinematic solution $(9.7,8.8$, 26.8) $\mathrm{mm}$ in the North, East and Up components. Compared with GPS only, the East and the Up components of GALILEO static solution are still less accuracy than $1 / 2$. Meanwhile, the accuracy of GALILEO's kinematic position is not much different from GPS.O 